\title{
Near-field characterization of Bragg mirrors engraved in surface plasmon waveguides
}

\author{
Jean-Claude Weeber, Yvon Lacroute, Alain Dereux \\ Laboratoire de Physique de l'Université de Bourgogne, UMR CNRS 5027, \\ 9 avenue Alain Savary, BP 47870, F-21078 Dijon, France
}

Eloïse Devaux, Thomas Ebbesen

Laboratoire des Nanostructures, ISIS,

8 allée Gaspard Monge, BP 70028, F-67083 Strasbourg, France

\author{
Christian Girard \\ Centre d'Elaboration des Matriaux et d'Etudes Structurales, UPR CNRS 8011, \\ 29 Rue J. Marvig, BP 4347, F-31055 Toulouse, France \\ María Ujué González, Anne-Laure Baudrion \\ Laboratoire de Physique de l'Université de Bourgogne, UMR CNRS 5027, \\ 9 avenue Alain Savary, BP 47870, F-21078 Dijon, France
}

(April 12, 2010)

\begin{abstract}
Surface plasmon waveguides (SPW's) are metal ridges featuring widths in the micrometre range and thicknesses of a few tens of nanometres. A focused ion beam (FIB) has been used to carve micro-scatterers into gold SPW's and the near-field distributions around these micro-structures are observed by means of a Photon Scanning Tunneling Microscope (PSTM). On the basis of near-field images, we show that a finite length periodic arrangement of
\end{abstract}


narrow slits can reflect a surface plasmon mode propagating along a SPW. The reflection efficiency of the micro-grating is found to depend upon the number of slits, the period of the grating and the incident wavelength. The optimum reflection efficiency is obtained for a period of the micro-grating equal to half the incident wavelength in vacuum. The PSTM images of the plasmon mirrors taken at different wavelengths allow us to measure the experimental dispersion curve of the SPW in the near-infrared. From this dispersion curve, we found that, in analogy with a surface plasmon (SP) excited on extended thin films, the group velocity of a SPW mode is close to the speed of light. For a given frequency in the near-infrared, the effective index of the SP mode supported by a $2.5 \mu \mathrm{m}$ wide SPW is also found to be significantly larger than the effective index of an extended thin film SP. Finally, we show that the optical properties of micro-gratings engraved into a SPW can be qualitatively approached by a standard Bragg mirror model.

07.79.Fc Near-field scanning optical microscopes

42.79.Gn Optical waveguides and couplers

73.20.Mf Collective excitations (including plasmons and other charge-density excitations)

78.66.-w Optical properties of specific thin films, surfaces, and low- dimensional structures 


\section{INTRODUCTION}

A polariton is an electromagnetic mode related to the oscillations of the polarization density. At the interface between two media such that the real parts of their frequency dependent dielectric functions have opposite signs, surface polariton featuring a field distribution that decays exponentially in both media may occur according to a well-known dispersion relation [1]. In the case where the material displaying a negative real part of the dielectric function is a metal, the polarization density oscillation corresponds to oscillations of the electron gas. The surface polariton is then called a Surface Plasmon Polariton (SPP) [2,3]. The electromagnetic field associated with a SPP can propagate along the interface to which it is bounded and decreases exponentially in the direction perpendicular to this interface. This vertical confinement makes SPP's appealing for the design of optical devices integrated in coplanar geometry. Until now, two features have hindered the development of SPP based integrated optics. First, the damping of the electron collective excitations limits the propagation distances. Second, very little is known about systems able to control the propagation of SPP.

In order to achieve efficient SPP guiding, two different approaches are currently investigated. The first kind of systems relies on the propagation of SPP modes along finite width thin metal films. When buried in an homogeneous dielectric medium, these surface plasmon waveguides (SPW's) allow the propagation of so-called "long-range" SPP's over distances of several millimeters at telecommunication frequencies [4-6]. Such a range of propagation distances make SPW's a possible alternative to high refractive index dielectric waveguides for the fabrication of miniaturized passive optical components such as splitters, filters or resonant cavities.

When deposited on a dielectric substrate, SPW's support SPP modes with effective propagation lengths of only few tens of microns in the near-infrared frequency range. Although in this case the propagation lengths are probably too small to be directly useful for integrated optical devices, this configuration offers the opportunity to observe SPP modes in 
direct space by near-field optical microscopy. For example, a strong lateral field confinement of SPP modes supported by silver SPW's has been demonstrated on the basis of Photon Scanning Tunneling Microscope (PSTM) images [7]. More recently, the PSTM images of gold SPW have shown that the modal field distributions of SPW modes are created by the coupling of finite width interface modes and boundary modes supported by the abrupt edges of the metal strips [8].

The second kind of devices allowing both the lateral confinement and the propagation control of SPP's are based on the interaction of surface plasmon with gratings formed with individual scatterers. Recently, SPP propagation along lines of defects created into a socalled "plasmonic crystal", such as an array of gold nano-particles deposited on a gold thin film, has been reported [9]. Similarly, silica posts texturing an extended silver thin film have been found to be very efficient to achieve the reflection of a locally launched surface plasmon [10]. Most of the previous works dealing with the scattering of SPP by periodic nano-structures have considered surface plasmons excited on extended thin films, i.e. thin films with lateral dimensions much larger than the wavelength of the surface wave.

In this work, we consider metal strips with typical widths of only a few wavelengths of the SPW modes and we study the interaction of these SPW modes with micro-structures engraved by Focused Ion Beam (FIB) milling into the metal strips. Specifically, we consider gratings made by a finite number of narrow slits texturing a $2.5 \mu \mathrm{m}$ wide SPW to show that these micro-gratings can be used as SPP mirrors. The paper is organized as follows. After a brief description of the experimental setup and the sample fabrication process in section II, we analyze in section III the PSTM images of a micro-grating recorded at different observation distances. The back-reflection of a SPW mode by a micro-grating is then discussed on the basis of these results. In section IV, we study the reflection efficiency of the micro-gratings as a function of different structural parameters such as the number of slits or the period of the gratings. Section $\mathrm{V}$ is dedicated to the measurement of the phase constant and the group velocity of a $2.5 \mu \mathrm{m}$ wide SPW using PSTM images obtained at different incident wavelengths in the near-infrared frequency range. Finally, we show in section VI 
that a standard Bragg mirror can model, at least qualitatively, the optical properties of the micro-gratings acting on SPW. The parameters of the equivalent Bragg mirror are deduced empirically from the experimental results of the previous sections.

\section{EXPERIMENTAL BACKGROUND}

Figure 1 shows the scanning electron microscope images of a typical structure considered in this study. The fabrication of the samples begins with an electron beam lithography process performed on an Indium Tin Oxide (ITO) doped glass substrate previously spin-coated with a layer of polymethylmethacrylate (PMMA) (200 nm thick). After the lithography stage, the exposed PMMA regions are dissolved and the sample is subsequently coated with a thermally evaporated gold $55-\mathrm{nm}$-thick film. This metal film is then lifted-off by dissolving the non-exposed PMMA areas, in such a way that after the lift-off process the sample consists of gold patterns deposited on the ITO-doped glass substrate (see Fig. 1(a)). The micro-structuration of these gold patterns is then performed by using a Dual Beam Strata 235 Focused Ion Beam (FIB). The microstructures we consider in this work consist of gratings made of a finite number of narrow slits (150-nm-wide) engraved into $2.5 \mu \mathrm{m}$-wide gold strips (Fig. 1).

To perform the optical excitation of the microstructured SPW, the sample is first attached with an index matching fluid to the hypotenuse of a right-angle glass prism. A surface plasmon is then locally launched in the Kretschmann-Raether configuration by means of a focused beam. As shown schematically on Fig. 1(a), the incident spot is located on the homogeneous thin film area to which the SPW's are connected. Thus, the SPW's excitation is performed by coupling with an extended thin film surface plasmon and not by direct illumination. An efficient coupling between the thin film surface plasmon and the SPW mode supported by a metal strip is achieved if the incident spot is located in front of the strip and if the plane of incidence is parallel to its axis.

The PSTM setup used in this work has been previously described in detail [7]. The PSTM 
relies on the frustration of evanescent fields using a local probe attached to a piezo-tube and scanned in the close vicinity of the sample surface [11]. In our device, the local probes are multi-mode optical fiber tips coated with a thin layer of chromium $(10 \mathrm{~nm})$ in order to prevent that a significant amount of radiative light enters the fiber and contributes to the near-field optical signal [12]. The metal coating also allows the detection of a tunnel current when a bias voltage is applied between the metal coated tip and the conductive surface of the sample. The detection of such a tunnel current is used to define the origin for the measurement of the absolute tip-sample distance since it occurs for the tip in quasi-contact with the sample surface.

\section{SPW MODE BACK-REFLECTION BY A MICRO-GRATING}

Two recent experimental studies have shown that either the abrupt discontinuity ending the extremity of a metal strip [7] or a single narrow groove engraved in an extended thin silver film [13] can efficiently reflect a SPP. In order to show the effect of the presence of a micro-grating on the propagation of a SPW mode, we show in figure 2(a) a large scan PSTM image recorded over a mirror consisting of ten slits milled in a $2.5 \mu \mathrm{m}$ wide SPW. The period of the micro-grating is $400 \mathrm{~nm}$ and the incident light frequency corresponds to a wavelength in vacuum of $\lambda_{0}=800 \mathrm{~nm}$. The micro-grating is located at a distance of 15 $\mu \mathrm{m}$ from the output end of the triangularly shaped area (named taper in the following) connecting the strip to the extended thin film. Well pronounced interference fringes with

a period of roughly $\frac{\lambda_{0}}{2}$ are visible all along the SPW while a two-dimensional standing wave pattern is observed over the taper. A longitudinal cross-cut of the optical image is displayed in figure 2(b) and shows more in detail the near-field intensity distribution over the the SPW. In analogy with a surface plasmon mode supported by an extended thin film propagating towards positive values of $y$, the electromagnetic field associated with a SPW mode is expected to depend upon $y$ as $\exp (-\alpha+i \beta) y$ where $\alpha$ and $\beta$ denote respectively the attenuation and the phase constant of the surface plasmon. However, it can observed on 
fig. 2(b) that the intensity of the SPW mode does not decrease exponentially as it propagates along the metal strip. Indeed, the optical signal at the center of the SPW increases from the end of the taper (corresponding to $y=4.0 \mu \mathrm{m}$ on fig. $2(\mathrm{~b})$ ) to $y=11 \mu \mathrm{m}$. Furthermore, the transverse intensity distribution changes dramatically with $y$ over that range as shown by the two optical profiles displayed respectively in fig. 2(c) and 2(d). Close to the SPW input ( fig. 2(c)), the optical profile exhibits a three peaks structure while a large central peak flanked by two shoulders is observed when the SPW mode has propagated over few microns (fig. 2(d)). Since it has been shown in a previous study that the SPW modes are hybrid modes, also called "super-modes", resulting from the superposition of finite size interface modes and boundary modes supported by the edges of the metal strip [8], we can thus assume that the spatial transient we observe on the PSTM image is due to the fact that these different modes must interact over a distance long enough in order to build-up the SPW mode.

As shown in fig. 2(b), the near-field intensity vanishes after the micro-structures leading to the conclusion that the incident SPW mode is mostly back-reflected and scattered by the grating. The standing wave pattern visible on the PSTM image could then arise from the interference of the incident SPP with either a back-reflected SPP or a scattered wave. In the near-infrared frequency range, we can reasonably assume that, in analogy with an extended thin film SPP, the phase constant of the SPW mode is close to the incident light wave-vector modulus in vacuum, $k_{0}=\frac{2 \pi}{\lambda_{0}}$. Therefore, from the momentum conservation law is derived that the micro-mirror could generate a scattered field propagating at grazing incidence regarding that in this experiment the period of the grating is equal to $\frac{\lambda_{0}}{2}$. Moreover, taking the phase constant of the incoming SPW mode close to $k_{0}$, the interference of the incident SPP with either a back-reflected SPP or a contra-propagating scattered wave traveling at grazing incidence could create a standing wave pattern with a period close to $\frac{\lambda_{0}}{2}$ in agreement with the rough estimate of the oscillations period observed on the PSTM image. In order to discriminate the origin of the standing wave pattern, we consider successively this two situations. 
If we suppose that the standing wave pattern arises from the interference of two contrapropagating SPP's traveling respectively toward the positive and negative values of $y$, then along a line $x=$ constant located at a distance $z_{0}$ from the air/metal interface supporting the SPP's, the intensity of each component $\delta$ of the electric field is given by:

$$
\begin{array}{r}
I_{\delta}\left(y, z_{0}\right)=E_{\delta, 0}^{2} \exp \left(-2 \alpha y-2 k_{z} z_{0}\right)+E_{\delta, r}{ }^{2} \exp \left[2 \alpha\left(y-y_{m}\right)-2 k_{z} z_{0}\right] \\
+2 E_{\delta, 0} E_{\delta, r} \exp \left(-\alpha y_{m}\right) \exp \left(-2 k_{z} z_{0}\right) \cos (2 \beta y+\phi)
\end{array}
$$

where $\alpha$ and $\beta$ are respectively the attenuation and the phase constant of the SPP. $E_{\delta, 0}$ and $E_{\delta, r}$ are the incident and the reflected amplitudes of the component $\delta$ of the electric field and $y_{m}$ denotes the location of the mirror. The $k_{z}$ component is the positive imaginary part of $\sqrt{k_{0}^{2}-(\beta+i \alpha)^{2}}$ and $\phi$ is the dephasing at reflection. For the interference of an incident SPP and a contra-propagative radiative plane wave traveling parallel to the metal/air interface, the intensity of the electric field can be written as:

$I_{\delta}\left(y, z_{0}\right)=E_{\delta, 0}^{2} \exp \left(-2 \alpha y-2 k_{z} z_{0}\right)+E_{\delta, r}^{2}+2 E_{\delta, 0} E_{\delta, r} \exp \left(-k_{z} z_{0}\right) \exp (-\alpha y) \cos \left[\left(\beta+k_{0}\right) y+\psi\right]$

where $\psi$ is a dephasing depending upon $k_{0}$ and $y_{m}$. The contrast of the interference fringes is defined as:

$$
C=\frac{I_{\max }-I_{\min }}{I_{\max }+I_{\min }}
$$

where $I_{\max }$ and $I_{\min }$ correspond respectively to the electric field intensity at a given maximum and at the following minimum. Assuming that the damping distance of the SPP is large compared to the standing wave pattern period, the contrast of the interference fringes due to the two contra-propagating SPP can be obtained from equation (1) as:

$$
C_{s p-s p}(y)=\frac{2 E_{0} E_{r} \exp \left(-\alpha y_{m}\right)}{E_{0}^{2} \exp (-2 \alpha y)+E_{r}^{2} \exp \left(2 \alpha\left(y-y_{m}\right)\right)}
$$

If we consider now the interference of an incident SPP and a contra-propagating radiative wave traveling at grazing incidence, the contrast of the standing wave pattern can be written from equation (2) as: 


$$
C_{s p-r a d}\left(y, z_{0}\right)=\frac{2 E_{0} E_{r} \exp \left(-k_{z} z_{0}\right) \exp (-\alpha y)}{E_{0}^{2} \exp (-2 \alpha y) \exp \left(-2 k_{z} z_{0}\right)+E_{r}^{2}}
$$

Therefore, from (4) and (5), we can conclude that in the case of two contra-propagating SPP the contrast of the standing wave pattern does not depend on $z_{0}$ while this dependence does exist for the interference of an incident SPP and a radiative wave traveling at grazing incidence. In fact, if we assume that the reflected amplitude $E_{r}$ is small compared to the term $E_{0}^{2} \exp (-2 \alpha y) \exp \left(-2 k_{z} z_{0}\right)$, then the contrast $C_{s p-r a d}$ increases exponentially with $z_{0}$.

So, to elucidate the origin of the standing wave pattern observed on the PSTM image, we have recorded PSTM images of the micro-structured strip for different tip-sample distances. The images corresponding to $z_{0}=340 \mathrm{~nm}, 280 \mathrm{~nm}$ and $170 \mathrm{~nm}$ are shown respectively on figures 3(b), (c) and (d). The transverse cross-cuts of each image are displayed in figure 3(e). For the smallest tip-sample distance, the intensity distribution exhibits the three peaks structure characteristic of a $2.5 \mu \mathrm{m}$-wide gold strip illuminated with an incident wavelength in vacuum of $800 \mathrm{~nm}$ [8]. The longitudinal optical profiles corresponding to each value of $z_{0}$ are also shown in figure $3(\mathrm{f})$. By averaging the contrast of up to ten oscillations in each profile, we have found that the average contrast $C_{\text {avg }}$ equal to $0.30,0.32$ and 0.30 for $z_{0}=$ $170 \mathrm{~nm}, 280 \mathrm{~nm}$ and $340 \mathrm{~nm}$ respectively. Thus, the experimental average contrast of the standing wave pattern does not depend significantly on the tip-sample distance although the near-field intensity at a given observation point is divided by a factor of about 5 when increasing $z_{0}$ from $170 \mathrm{~nm}$ to $340 \mathrm{~nm}$ (see figure 3(f)). From this result, we conclude that the standing wave pattern observed on the PSTM images is created by the interference of two contra-propagating SPP modes traveling along the metal strip.

\section{REFLECTION EFFICIENCY OF THE MICRO-MIRRORS}

The results of the previous section demonstrate the ability of a micro-grating to reflect a SPW mode. With the aim of characterizing the reflection process by such a mirror, we investigate in this section the influence on the reflection efficiency of structural parameters such as the number of slits or the period of the micro-gratings. 


\section{A. Influence of the number of slits}

As mentioned above, a single slit engraved in a metal film can reflect a surface plasmon [13]. It is then possible that the reflection efficiency of the ten slits micro-grating considered in the previous paragraph could be obtained as well with mirrors consisting of a reduced number of slits $\left(N_{s}\right)$. In order to study the evolution of the reflection efficiency with $N_{s}$, we have designed samples with one, three, five and ten slits. For all these samples, the mirrors are located at a distance of $15 \mu \mathrm{m}$ from the input of the $2.5-\mu \mathrm{m}-$ wide SPW, the width of the slits and the period of the gratings being $150 \mathrm{~nm}$ and $400 \mathrm{~nm}$ respectively. The optical profiles recorded along an observation line located over the center of each micro-structured SPW are displayed in figure 4. The interference of the incident SPW mode with the field scattered by a single slit engraved into the metal strip (see fig. 4(a)) creates a pattern of non-periodic large amplitude oscillations extending over a distance of more than $4 \mu \mathrm{m}$. A very low contrast standing wave pattern which can be attributed to a weak back-reflection of the incident SPP, is also visible on the left part of the optical profile. In spite of the back-reflection and the scattering, the incident SPP is also transmitted through the slit. Indeed, a rather large near-field intensity is still detected for the observation points located after the slit $(y>15 \mu \mathrm{m})$. Since the slit engraved into the SPW consists of an air gap separating two parts of the metal strip and each part of the metal strip can support a SPP while no SPP can propagate in air, the transmission of the SPW mode through the slit can then be viewed as a kind of tunnel effect.

For an increasing number of slits, the amplitude of the SPP transmitted through the micro-grating vanishes while conversely the contrast of the standing wave pattern increases. The average contrast $C_{\text {avg }}$ computed over more than 20 oscillations of the different standing wave patterns is plotted in figure $4(\mathrm{e})$ as a function of the number of slits. The contrast reaches an asymptotic value of about 0.26 for the micro-grating consisting of ten slits. Note that the contrast increases between $N_{s}=5$ and $N_{s}=10$, leading to the conclusion that the five last slits of the micro-grating with $N_{s}=10$ do contribute to the back-reflection of 
the incident SPP. In other words, the hopping of the incident SPP over the successive slits of the micro-grating is efficient enough to allow the propagation of the SPP through a micro-structured area with a length of several microns.

The increase of the standing wave pattern contrast is obviously related to a corresponding increase of the reflection efficiency of the mirror. However, without a reliable modeling of the metal strip SPP modes, we can only roughly estimate these efficiency. Considering an incident SPP propagating at a gold/air interface and a mirror located at $15 \mu \mathrm{m}$ from the launch site, we can compute the reflection coefficient of the mirror needed for matching the value of the contrast of the theoretical standing wave pattern with the contrast of the experimental optical profiles. We define the reflection coefficient of the mirror by $r=E_{\delta}^{r} / E_{\delta}^{0}$ where $E_{\delta}^{r}$ and $E_{\delta}^{0}$ denote respectively the amplitude of the reflected and the incident $\delta$ component of the SPP electric field at the location of the mirror. Assuming a similar reflection coefficient for the transverse and the longitudinal components of the SPP electric field, a contrast for the computed standing wave pattern of 0.26 is obtained with $r$ equal to $25 \%$.

\section{B. Influence the micro-grating period}

Up to now, we have only considered micro-gratings with a period $P=400 \mathrm{~nm}$. With the aim of studying the influence of the period on the reflection efficiency, we consider now mirrors with three different periods $(350 \mathrm{~nm}, 400 \mathrm{~nm}$ and $500 \mathrm{~nm}$ ) while keeping a fixed number of ten slits engraved at a distance of $15 \mu \mathrm{m}$ from the SPW input. The longitudinal optical profiles shown in the right part of figure 5 have been recorded over these samples for two incident wavelengths $\lambda_{0}=753 \mathrm{~nm}$ (solid line) and $\lambda_{0}=800 \mathrm{~nm}$ (dashed line) (The PSTM images recorded with $\lambda_{0}=800 \mathrm{~nm}$ are shown in the left part of figure 5). A neat standing wave pattern is observed for the mirrors with $P=350 \mathrm{~nm}$ and $P=400 \mathrm{~nm}$ while mainly the scattering on the mirror is visible when $P=500 \mathrm{~nm}$. The low contrast standing wave patterns in figure $5(\mathrm{f})$ indicate that, for both wavelengths, the mirror with a period 
of $500 \mathrm{~nm}$ reflects very poorly the incident SPW mode. The standing wave pattern being much more pronounced for $P=350 \mathrm{~nm}$ and $P=400 \mathrm{~nm}$, it is worth to notice that the highest contrast is obtained for $\lambda_{0}=753 \mathrm{~nm}$ if $P=350 \mathrm{~nm}$ and for $\lambda_{0}=800 \mathrm{~nm}$ if $P=400 \mathrm{~nm}$. In order to investigate more in detail the efficiency of the mirror when the ratio $P / \lambda_{0}$ is close to 0.5 , the micro-grating with a period $P=400 \mathrm{~nm}$ has been imaged at six different wavelengths while only two have been used for $P=350 \mathrm{~nm}$ and $P=500 \mathrm{~nm}$. The average contrast of the standing wave-pattern obtained for the different period-wavelengths combinations is plotted as a function of the ratio $P / \lambda_{0}$ in figure 6 . The curve displayed in figure 6 shows that the contrast reaches a maximum when the incident wavelength in vacuum is twice the period of the micro-grating. The highest reflection efficiency of the mirror is expected for the SPP's back-reflected by each slit being in phase then we conclude that the effective wavelength of the SPP mode traveling through in the micro-structured area is very close to $\lambda_{0}$.

\section{Influence of the incident wavelength}

By changing the period of the micro-grating, we have found that the maximum reflection efficiency of the mirror is obtained for $P / \lambda_{0}$ equal to 0.5 . To further confirm this result, we have performed the PSTM imaging of a micro-grating with a fixed period of $400 \mathrm{~nm}$ by using incident wavelengths distributed over the whole tunability range of our Titanium-sapphire laser $(750 \mathrm{~nm}-830 \mathrm{~nm})$. In this case, the mirror consists of ten slits engraved at a distance of $25 \mu \mathrm{m}$ from the input of the $2.5 \mu \mathrm{m}$ wide SPW. A typical PSTM image of this mirror obtained with $\lambda_{0}=800 \mathrm{~nm}$ is shown in figure 7(a). As expected, a well pronounced standing wave pattern is visible on the near-field image. A cross-cut of this image taken along the axis $x=0$ is shown in figure $7(\mathrm{~b})$ (solid line). Unlike the standing wave pattern resulting from the interference of two contra-propagating SPP's (see equation (1)), we observe that the amplitude of the oscillations decreases dramatically when the observation point approaches the mirror. In fact, according to equation (2), such a damping occurs when an incident SPP interferes with a radiative wave. However, we have shown in section III that the 
standing wave pattern in the PSTM images of the micro-gratings arises from surface-waves interferences. Furthermore, unlike what is expected when a SPP interferes with a radiative wave, the amplitude of the oscillations in figure $7(\mathrm{~b})$ is not damped monotonically with the propagation distance. Indeed, it is found to be rather constant for $y<5 \mu \mathrm{m}$ (see dashed line in figure $7(\mathrm{~b})$ ). From these observations, we deduce that the damping of the standing wave pattern amplitude is not due to the interference of a SPP and a radiative wave but on the other hand, such a damping is not expected for two contra-propagating SPP's. To overcome this apparent contradiction, it is worth to notice that in our case we are not considering SPP excited on an extended thin film but the hybrid SPW modes. The damping could then be related to the intrinsic properties of these modes, i.e the back-reflected hybrid SPW mode has to propagate over a minimal "threshold" distance in order to arise as the result of the reflection, produced by each slit of the mirror, of each SPP component involved in the incident hybrid SPW. In the absence of a reliable modeling of these modes, this hypothesis is difficult to verify. However, it is consistent with the fact that, at the input of the SPW, such a spatial transient is necessary to operate the transition from the incident extended thin film SPP and the SPW mode (see fig. 2). Note also that the damping of the standing wave pattern is less pronounced when the mirror is engraved at a smaller distance from the SPW input (see for example fig 4(d)) indicating that the field distribution of the incident SPW mode at the location of the mirror influences the back-reflection process.

Although not fully understood, the damping of the standing wave pattern oscillations is not an obstacle to the spectroscopic study of the mirror efficiency on the basis of the PSTM images. Indeed, one can see on figure $7(\mathrm{c})$ that the contrast of the oscillations is rather constant for observation points located at a distance large enough from the mirror. Thus, by averaging this contrast over the five first micrometers $(y<5 \mu \mathrm{m})$ of each optical profile recorded with different incident wavelength, we obtain the curve plotted in figure 8 . The fitting of the experimental data exhibits a parabolic shape with a maximum corresponding to an incident wavelength in vacuum of $\lambda_{0}=800 \mathrm{~nm}$. Thus, in agreement with the previous experiment, we find that the maximum reflection efficiency is obtained for the wavelength 
$\lambda_{0}$ equal to twice the period of the micro-grating. This result suggests once again that the phase constant of the field traveling through the micro-structured area is very close to $k_{0}=\frac{2 \pi}{\lambda_{0}}$.

\section{DISPERSION CURVE OF THE SPW MODE}

The integration of micro-mirrors in SPW may have interest for SPP routing purposes for example, but beyond these practical applications micro-mirrors can also be used to characterize the fundamental physical properties of metal strip SPP. The general form of the electric field of a SPW mode propagating along the $y$ axis can be written as:

$$
\vec{E}(x, y, z)=\vec{E}_{0}(x, z) \exp (-\alpha+i \beta) y
$$

where $\alpha$ and $\beta$ denote respectively the attenuation and the phase constant of the SPP. The PSTM images of the micro-structured SPW's allow the measurement of these two constants simultaneously. Indeed by averaging up to 60 columns of a $512 \times 512$ pixels PSTM SPW image (see for example figure $7(\mathrm{a})$ ), a mean longitudinal optical profile can be obtained. From such a profile, one can measure the period $d$ of the standing wave pattern and the $1 / e$ damping distance $L_{s p}$ of the background intensity and then compute the attenuation and the phase constants according to the relations $\alpha=1 /\left(2 L_{s p}\right)$ and $\beta=\pi / d$.

The reflection efficiency of the micro-mirror is large enough over the tunability range of our laser to provide a well-defined standing wave pattern that allows an accurate measurement of the period $d$ and thus the computation of the phase constant $\beta$. The experimental dispersion curve of the SPW mode phase constant is shown on figure 9. The light line defined by $\beta=\omega / c$ and the dispersion curve of an extended gold/air interface surface plasmon are also displayed on this figure. The phase constant of the surface plasmon has been computed according to the well-known relation $\beta_{S P}=\Re\left(\frac{\omega}{c} \sqrt{\frac{\varepsilon(\omega)}{\varepsilon(\omega)+1}}\right)$ where $\Re$ and $\varepsilon(\omega)$ denote respectively the real part and the complex dielectric function of gold [3]. The values of $\varepsilon(\omega)$ used for the computation of $\beta_{S P}$ are experimental values measured by ellipsometry. Over the 
frequency range considered, the complex refractive index of gold has been found to be given by $\hat{n}=0.32+i\left(8.66 \times \lambda_{0}-2.05\right)$, where $\lambda_{0}$ is the incident wavelength in vacuum expressed in micrometer. It can be seen on figure 9 that, at a given frequency, the phase constant of the SPW mode is significantly larger compared to the one of a SPP launched on an extended thin film. Note that, in spite of the absence of crossing between the two dispersion curves, an efficient coupling between the two kinds of surface plasmon is observed [8]. Such an efficiency must be related to an overlap of the two kinds of surface plasmon resonance due to their respective linewidths.

The group velocity, defined by $v_{g}=d \omega / d k$, is commonly interpreted as the average velocity of a pulse [14]. However, both the shape and the amplitude of a pulse can be affected by the propagation into a dissipative and/or dispersive medium such that the concept of group velocity may lose its physical significance in this situation [15]. Nevertheless, even for a dispersive medium, the group velocity remains physically meaningful if the duration of the pulse is sufficiently long such that its spectrum has a significant amplitude only over a very narrow range of frequency. Thus, at a given frequency, the local slope of the dispersion curve of a surface plasmon can still be interpret as a pulse velocity for long enough excitations. With this restriction in mind, we can measure the average velocity of a pulse propagating along a metal strip directly from the experimental dispersion curve shown in fig.(9). For example, at a frequency corresponding to a wavelength of $800 \mathrm{~nm}$ in vacuum, we found a group velocity of $v_{g}=2.9 \times 10^{8} \mathrm{~ms}^{-1}$. For this range of frequency, the group velocity of a plasmon polariton excited at the surface of an extended thin gold film is also very close to the speed of light. It is then worth to notice that surprisingly, unlike what has been found for the damping distance [16], the finite width of the metal strip (only three times the incident wavelength) does not influence significantly the group velocity of the SPW mode. 


\section{ANALOGY WITH A BRAGG MIRROR}

A standard Bragg mirror is a stack of layers made of materials with different optical refractive index. For the SPP modes propagating along a metal strip, the micro-grating creates a periodic refractive index contrast such that, to some extent, a micro-grating can be viewed as a SPP Bragg mirror. The goal of this last section is to determine empirically the structural parameters of an equivalent one-dimensional Bragg mirror (EBM) which models qualitatively the optical properties of the micro-gratings engraved in a SPW.

The EBM is supposed to be illuminated in normal incidence by a radiative wave with a wave-vector equal to the phase constant of the SPW mode. This phase constant is known from the experimental dispersion curve discussed in the previous section. The EBM is made of two materials (A) and (B) modeling respectively the air gap of the slits and the pieces of the metal strip located in between two successive slits (figure 10(a)). The thickness of each layer in the EBM is taken equal to the dimension of the corresponding structures in the micro-gratings. For example, for a micro-grating with a period $P=400 \mathrm{~nm}$, the thickness of materials (A) and (B) are respectively $150 \mathrm{~nm}$ (width of the slits) and $250 \mathrm{~nm}$ (inter-slit distance). The EBM is fully characterized if the optical refractive indexes of each material are specified. These indexes are determined empirically using the experimental results discussed previously.

We have shown in section III that the reflection efficiency of the micro-gratings saturates for a rather small number of slits. Indeed, the contrast of the standing wave pattern changes by only $15 \%$ when switching from a mirror with $N_{s}=5$ to $N_{s}=10$ (see fig. $4($ f)). This result arises from the dramatic damping of the incident SPP as it propagates through the microstructured area of the metal strip. In order to simulate this damping in the EBM model, the air gaps engraved in the SPW are modeled by an absorbing material (material (A)) with a complex refractive index $\hat{n_{A}}=n_{A}+i \kappa_{A}$. Unlike the air gaps, the metal strip pieces located between two successive slits can support a SPP mode and then are represented by a real refractive index material in the EBM model. By changing both the period of the 
micro-grating and/or the incident frequency, we have found that the maximum reflection efficiency of the mirror is obtained for an incident wavelength in vacuum equal to two times the period of the grating. As mentioned before, this suggests that the phase constant of the SPP field traveling through the mirror is very close to $k_{0}$. As a consequence, for the EBM model, the real part of refractive index of both materials (A) and (B) should be chosen very close to 1.0. Material (B) simulates a medium supporting a SPP. A SPP being an evanescent wave, it's phase constant $\beta$ is larger than $k_{0}$ leading to an effective index $n_{\text {eff }}=\beta / k_{0}$ larger than 1.0 for the medium supporting the SP. In order to satisfy the two aforementioned conditions, we chose an optical refractive index for material (B) equal to $n_{B}=1.01$. Since material (A) models the air gaps in the SPW, $n_{A}$ is taken equal to 1.0. In order to estimate the extinction coefficient of (A), the EBM reflectivity has been computed as a function of the number of layers $N_{s}$ of material (A) for a several values of $\kappa_{A}$. The curve plotted in figure 10 (a) has been obtained with $\kappa_{A}=0.3$. We found that, in qualitative agreement with the experimental curve of fig. $4(\mathrm{f})$, the reflectivity of the EBM saturates at $N_{s}=10$. The curve displayed in figure 10(b) shows the reflectivity of the EBM $\left(N_{s}=10\right)$ computed for frequencies corresponding to the tunability range of our laser. As expected, the maximum reflectivity is obtained for a wavelength $\left(\lambda_{0}=803 \mathrm{~nm}\right)$ almost equal to double the period of the EBM. The EBM reflectivity curve exhibits a parabolic shape which is one more time in qualitative agreement with the experimental results (fig. 8). The calculation of the EBM reflectivity has been performed with a refractive index of the input and output medium equal to 1.07 which is the effective index corresponding to the SPW mode as computed using the experimental dispersion curve shown in figure 9. It is then worth to notice that, in order to obtain a qualitative agreement with the experimental results, the SPP traveling inside the micro-structured area is modeled with an effective index $\left(n_{B}=1.01\right)$ different from the effective index of the SPW mode propagating along the homogeneous (non-structured) SPW although in both cases we consider SPP at a gold/air interface. A possible explanation for such a difference could be that, in analogy with localized surface plasmon of metallic nano-particles, the geometry of the system supporting the SPP plays a key role. For the 
homogeneous SPW, the SPP mode propagates along a metal strip with a length much larger that it's width. On the contrary, inside the micro-structured area, the SPP is supported by pieces of the metal strip with a dimension along the propagation direction much smaller than the SPW width.

With the aim of checking the reliability of our empirical model, we consider finally the influence of the period $P$ on the reflectivity of the EBM. Similarly to the experimental situation, we have simulated three different periods $P=350 \mathrm{~nm}, P=400 \mathrm{~nm}$ and $P=500$ $\mathrm{nm}$, by taking the inter-slit distance respectively equal to $200 \mathrm{~nm}, 250 \mathrm{~nm}$ and $350 \mathrm{~nm}$. The reflectivity of the EBM computed for the wavelengths considered experimentally for each period is plotted as a function of the ratio $P / \lambda_{0}$ in figure $10(\mathrm{~d})$. Although small differences with the experimental curve (fig. 6) can be observed, we found that, qualitatively, the EBM models accurately the influence of the period on the SPP back-reflection efficiency of the micro-gratings.

\section{CONCLUSION}

In summary, we have analyzed in detail the integration of micro-mirrors into surface plasmon waveguides. A PSTM has been used to map the near-field optical intensity distribution of a SPP propagating along a metal strips with a width of $2.5 \mu \mathrm{m}$ where finite length gratings of slits have been engraved by FIB milling. The PSTM images of the microgratings show a well pronounced standing wave pattern. This standing wave pattern has been found to arise from the interference of two contra-propagating SPP since the contrast of the interference fringes does not depend upon the distance between the observation plane and the surface of the sample.

By considering micro-gratings with an increasing number of periods, we have shown that the reflection efficiency of the mirrors saturates for a rather low number of about ten slits. This saturation is due to the dramatic damping of the incident SPW mode as it propagates through the micro-grating. Using three different periods, we have found that the optimum 
mirrors efficiency is obtained for an incident wavelength in vacuum equal to twice the period of the grating. This result has been confirmed by the spectroscopic study of the reflection efficiency performed over the whole tunability range of our laser $(753 \mathrm{~nm}-830 \mathrm{~nm})$ for a mirror with a period of $400 \mathrm{~nm}$.

An accurate measurement of the standing wave pattern period has been used to obtain the experimental dispersion curve of the SPW mode phase constant. On the basis of this dispersion curve, we have found that, in the near-infrared, the effective index of the SPW mode we consider is about $n_{e f f}=1.07$ and that the group velocity is close to the speed of light in vacuum. Finally, we have demonstrated that the optical properties of the micro-gratings integrated into SPW can be modeled, at least qualitatively, by mean of an equivalent Bragg mirror. From this analogy we conclude that, in principle, the micro-gratings implanted in metal strips act on SPW modes as Bragg mirrors although the SPP Bragg mirrors studied in this paper might have specific properties such as the influence of the micro-gratings geometry on the effective index of SPP propagating through the zone where the slits are engraved.

\section{ACKNOWLEDGMENTS}

This work was supported by the Regional Council of Burgundy (ARCEN project), by the French CNRS (STIC: EPML 20) and, in its final phase, by the European Commission (NoE contract FP6-IST-2002-1-507879). One of us (M. U. G.) acknowledges funding from the Spanish Ministry of Education. 


\section{REFERENCES}

[1] K. L. Kliewer and R. Fuchs, Adv. Chem. Phys. 27, 355 (1974).

[2] A. Otto, Zeit. für Physik 216, 398 (1968).

[3] H. Raether, Surface Plasmons, Springer Tracts in Modern Physics, Vol. 111 (Springer, Berlin, 1988).

[4] P. Berini, Phys. Rev. B 61, 10484 (2000).

[5] R. Charbonneau, P. Berini, E. Berolo, E. Lisicka-Shrzek, Optics Lett. 25, 844 (2000).

[6] T. Nikolajsen, K. Leosson, I. Salahutdinov, S. Bozhevolyi, Appl. Phys. Lett. 82, 668 (2003).

[7] J. C. Weeber, J. R. Krenn, A. Dereux, B. Lamprecht, Y. Lacroute, J.P. Goudonnet, Phys. Rev. B 64, 045411 (2001).

[8] J. C. Weeber, Y. Lacroute, A. Dereux, Phys. Rev. B 68, 115401 (2003).

[9] S.I. Bozhevolnyi, J. E. Erland, P. M. W. Skovgaard, J. M. Hvam, Phys. Rev. Lett. 86, 3008 (2001).

[10] H. Ditlblacher, J.R. Krenn, G. Schider, A. Leitner, F. R. Aussenegg, Appl. Phys. Lett. 81, $1762(2002)$.

[11] R. C. Reddick, R. J. Warmack, T. L. Ferrell, Phys. Rev. B 39, 767 (1989).

[12] C. Peeters, E. Flück, A. M. Otter, M.L.M. Balistreri, J.P. Korterik, L. Kuipers, N.F. van Hulst, Appl. Phys. Lett. 77, 142 (2000).

[13] J. Seidel, S. Grafström, L. Eng, L. Bischoff, Appl. Phys. Lett. 82, 1368 (2003)

[14] J. D. Jackson, Classical Electrodynamics, J. Wiley, New-York (1975).

[15] J. A. Stratton, Electromagnetic Theory, McGraw-Hill, New-York (1941). 
[16] B. Lamprecht, J.R. Krenn, G. Schider, H. Ditlbacher, M. Salerno, N. Felidj, A. Leitner, F.R. Aussenegg, J-C. Weeber, Appl. Phys. Lett. 79, 51 (2001). 


\section{FIGURES}
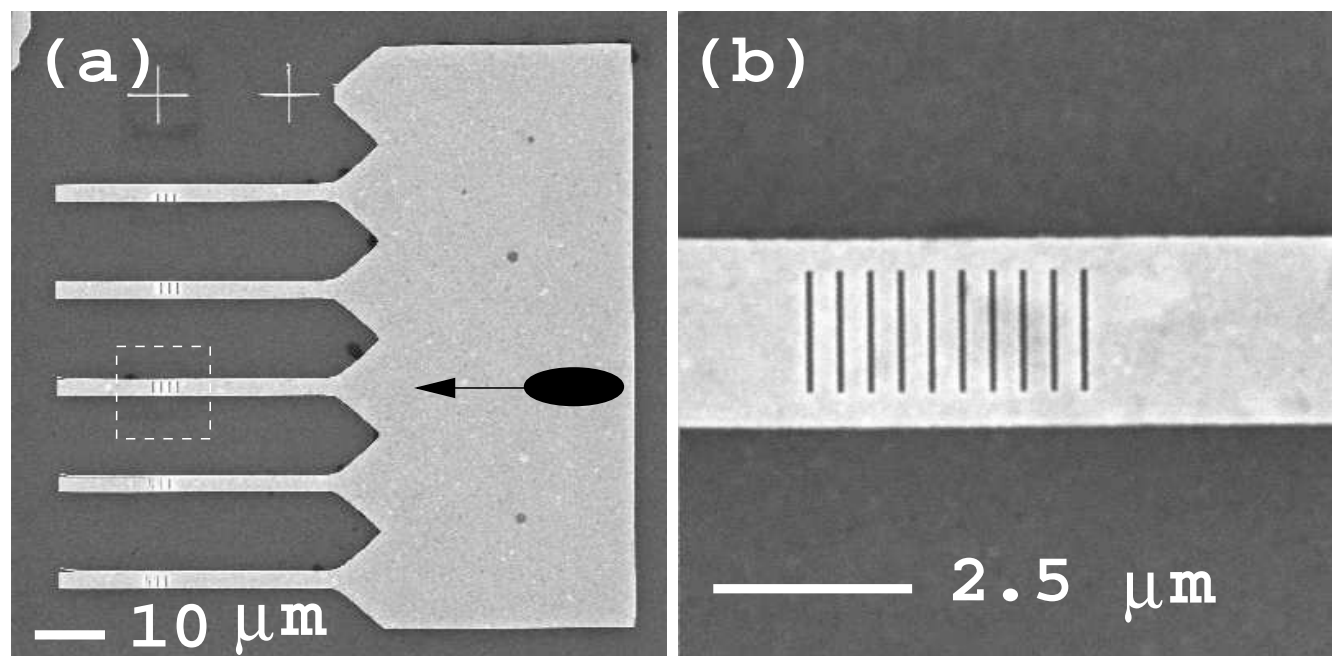

FIG. 1. Scanning electron microscope (SEM) images of a typical structure used in this study.

(a) The sample consists of a gold pattern (thickness $55 \mathrm{~nm}$ ) fabricated by electron-beam lithography and deposited on an indium-tin oxide doped glass substrate. The black ellipse shows the location of the totally reflected focused light beam (incident from the substrate) used for the excitation of the surface plasmon. (b) A FIB is used to a engrave micro-gratings into each metal strips. The micro-grating shown here consists of ten parallel slits of same width $(150 \mathrm{~nm})$. In this case, the period of the pattern is $400 \mathrm{~nm}$. 


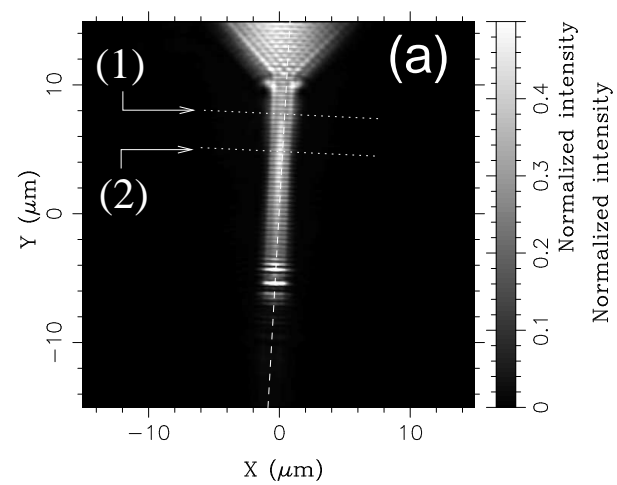

(b)
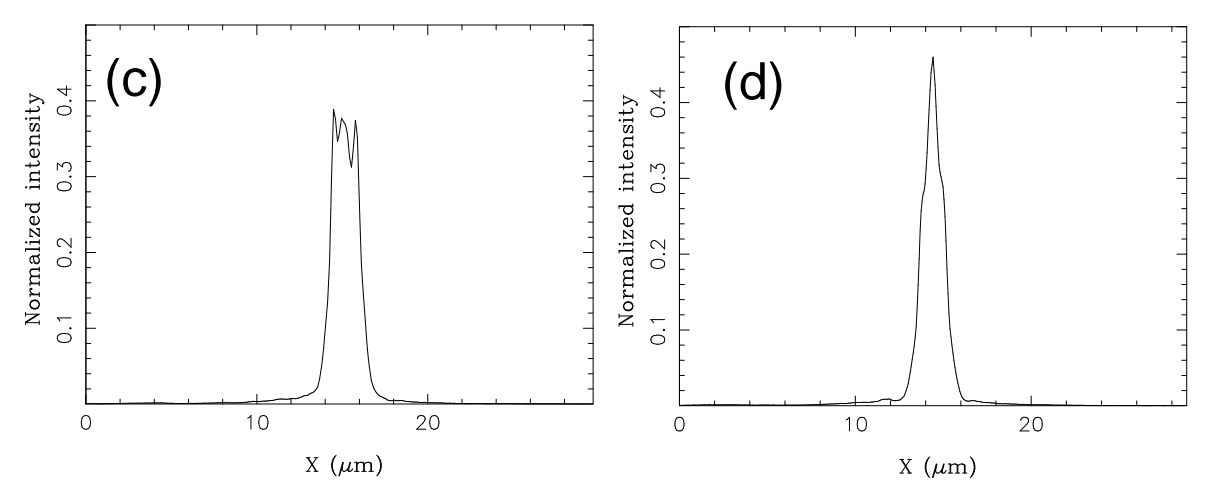

FIG. 2. (a) Large scan PSTM image of a micro-structured SPW. (b) Longitudinal cross-cut of the near-field image along the dashed line in (a). (c) and (d) Transverse cross-cuts of the PSTM image taken along the dotted lines (1) and (2) shown in (a) respectively. 

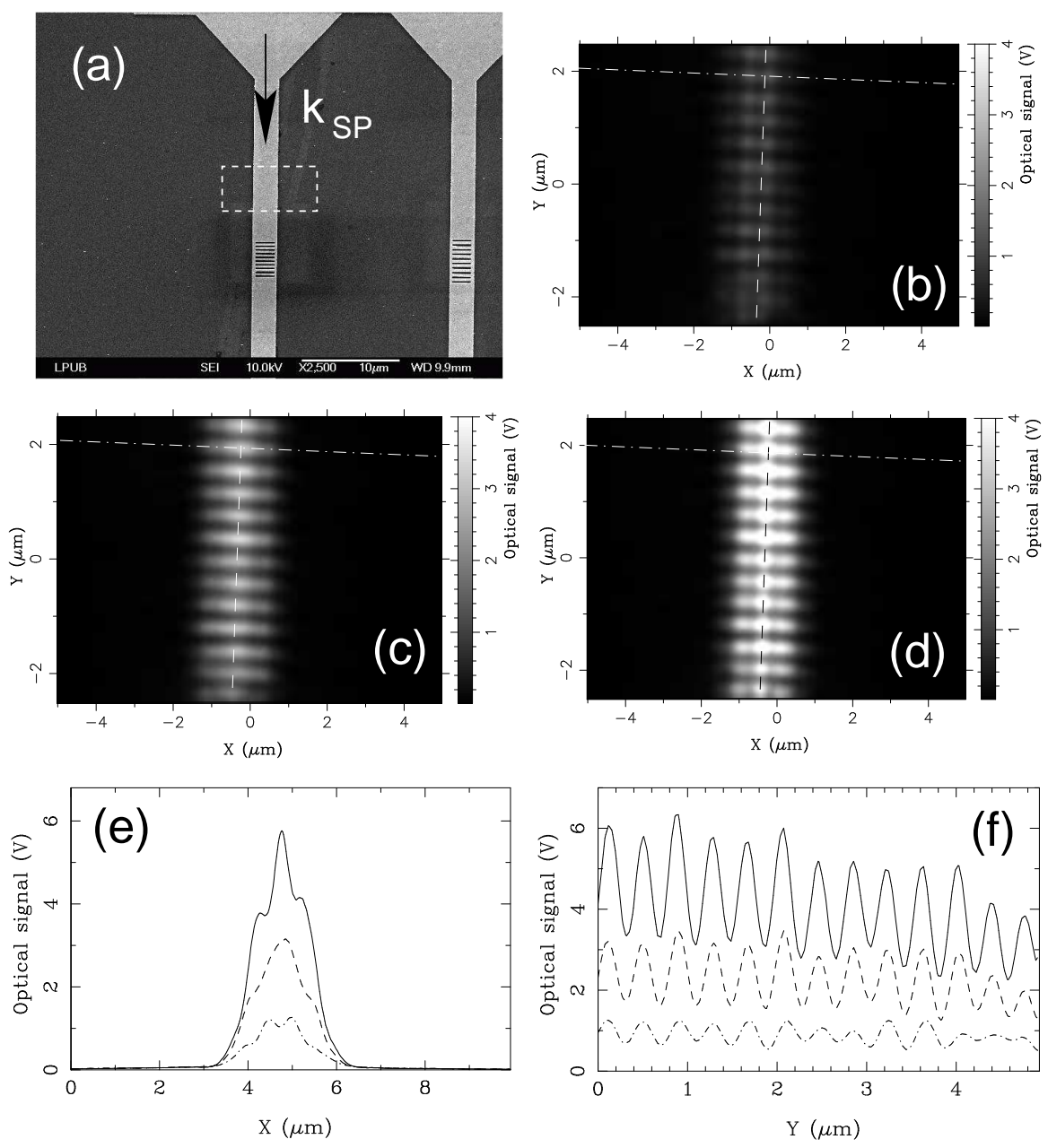

FIG. 3. (a) SEM image of the sample used to investigate the influence of the tip-sample distance on the standing wave pattern contrast. The incident wavelength is $800 \mathrm{~nm}$ and the period of the grating is $400 \mathrm{~nm}$. (b), (c), (d) Small scans PSTM images of the standing wave pattern obtained for tip-sample distances of respectively $340 \mathrm{~nm}, 280 \mathrm{~nm}$ and $170 \mathrm{~nm}$. The white dashed perimeter in fig. 3(a) shows approximately the scanning area. (e) Transverse cross-cuts of the PSTM images (solid: $170 \mathrm{~nm}$, dashed: $280 \mathrm{~nm}$, dashed-dotted:340 nm). (f) Longitudinal cross-cuts of the PSTM images (solid: $170 \mathrm{~nm}$, dashed: $280 \mathrm{~nm}$, dashed-dotted: $340 \mathrm{~nm}$ ). 

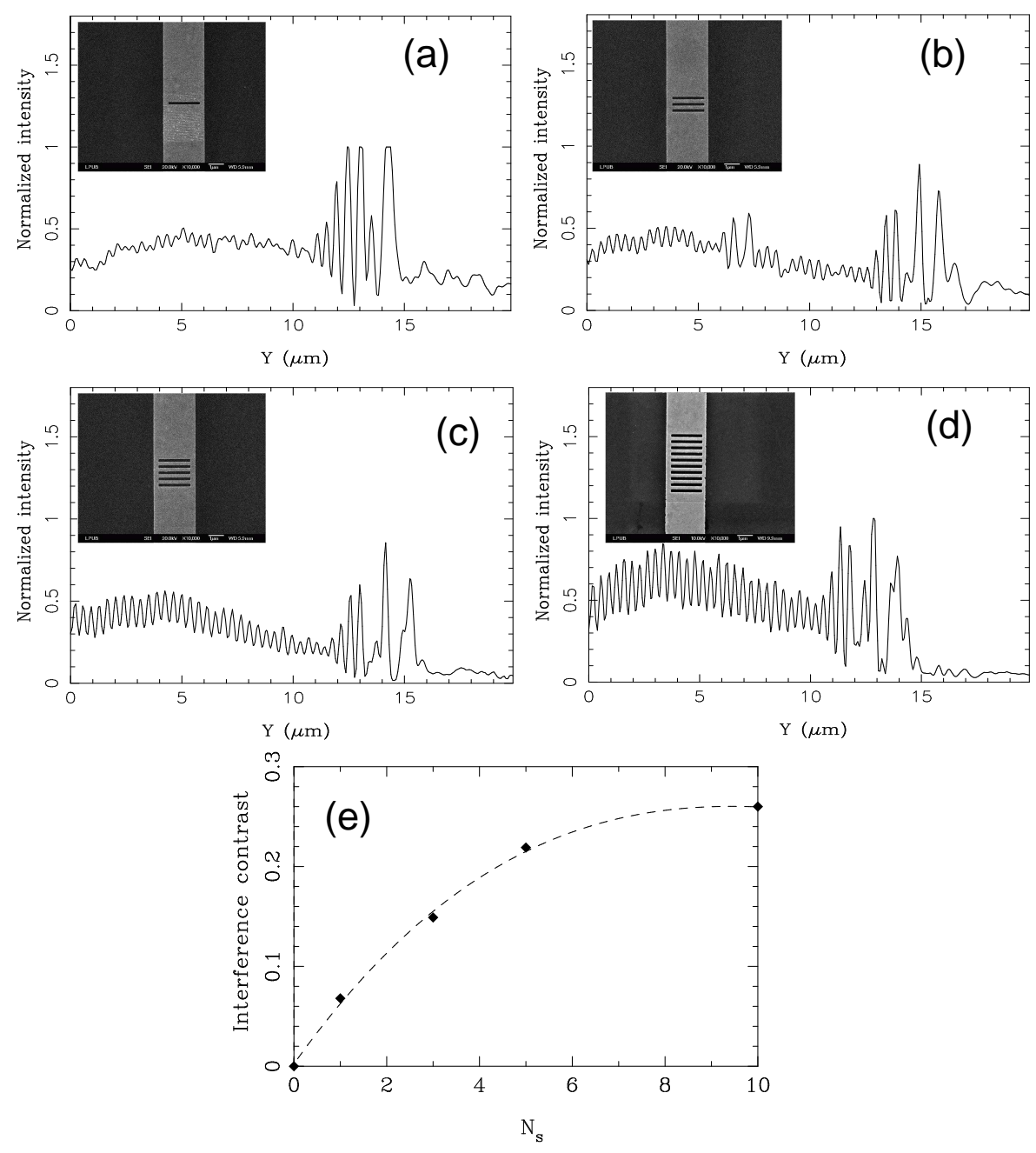

FIG. 4. PSTM optical profiles recorded over micro-gratings with an increasing number of slits $N_{s}$. (a) $N_{s}=1$, (b) $N_{s}=3$, (c) $N_{s}=5$, (d) $N_{s}=10$. (e) Plot of the standing wave pattern average contrast versus the number of slits, $N_{s}$. The dashed line is a least square polynomial fitting of the experimental values (dots). 

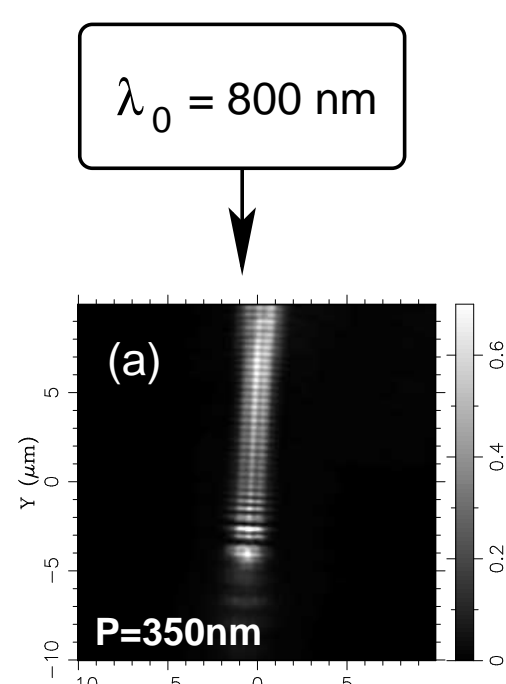

$\mathrm{X}(\mu \mathrm{m})$
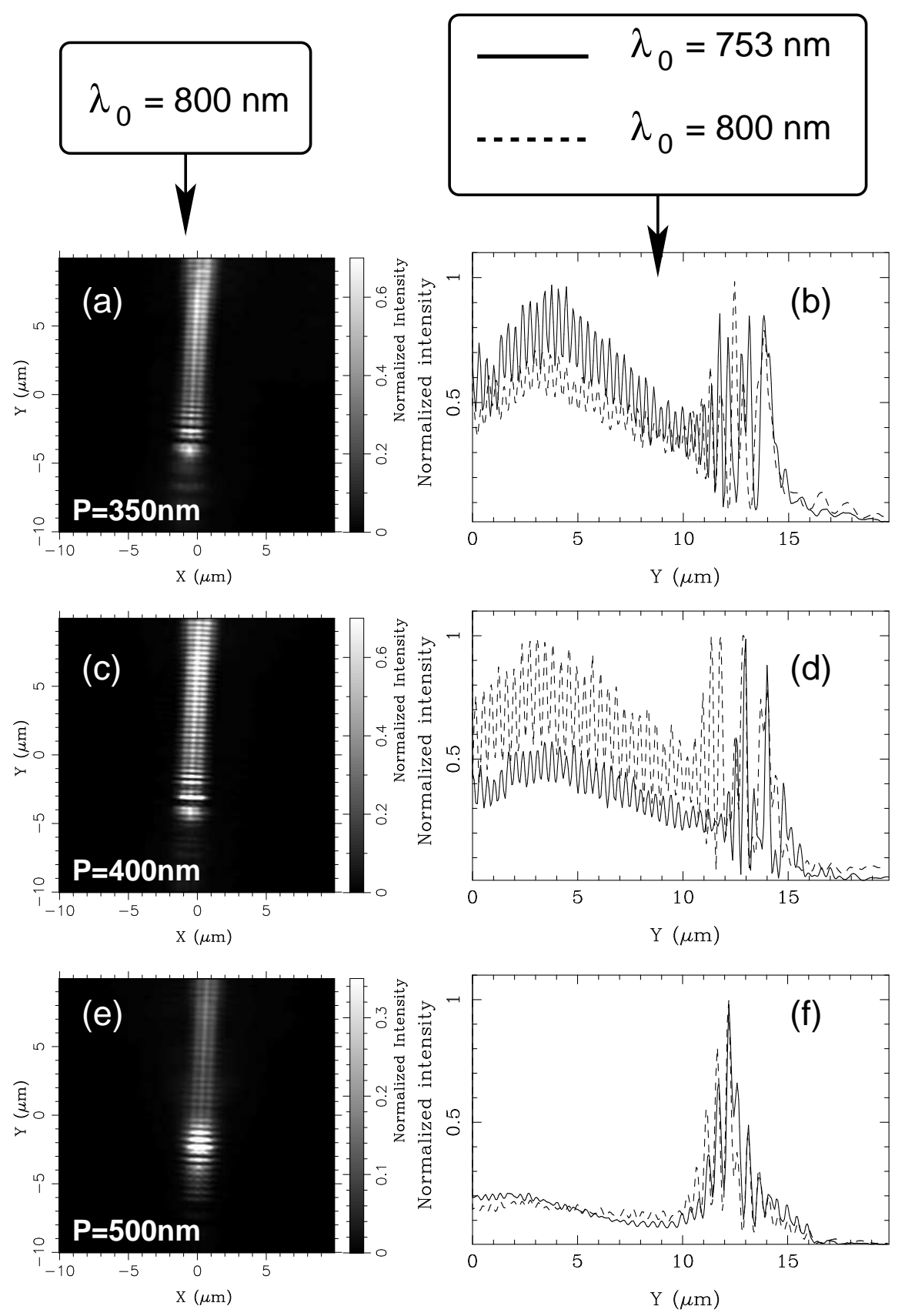

FIG. 5. (a), (c), (e) PSTM images of SPP propagating along a metal strip (width=2.5 $\mu \mathrm{m}$ ) textured by micro-gratings with different periods, $P=350 \mathrm{~nm}, 400 \mathrm{~nm}$ and $500 \mathrm{~nm}$. The incident wavelength in vaccum is $800 \mathrm{~nm}$. (b) (resp. (d),(f)) Cross-cut of the PSTM images of the micrograting with $P=350 \mathrm{~nm}$ ( resp. $P=400 \mathrm{~nm}, P=500 \mathrm{~nm}$ ). The dashed (resp. solid) line corresponds to an incident wavelength of $800 \mathrm{~nm}$ (resp. $753 \mathrm{~nm}$ ). 


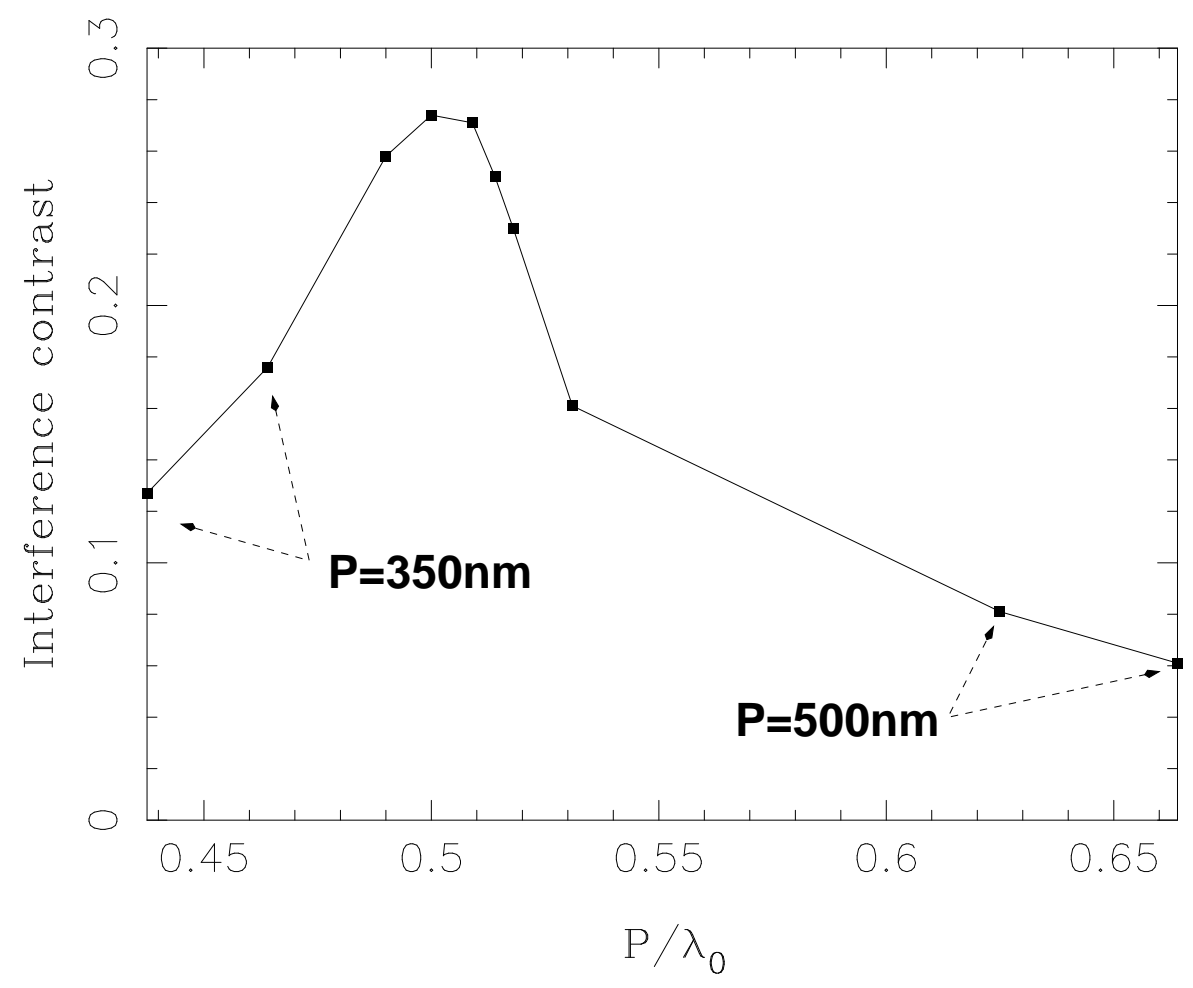

FIG. 6. Average contrast of the standing wave pattern versus the ratio of the period of the grating $(P)$ to incident wavelength in vacuum $\left(\lambda_{0}\right)$. The experimental points distributed around $P / \lambda_{0}=0.5$ are obtained with a micro-grating period $P$ of $400 \mathrm{~nm}$. 

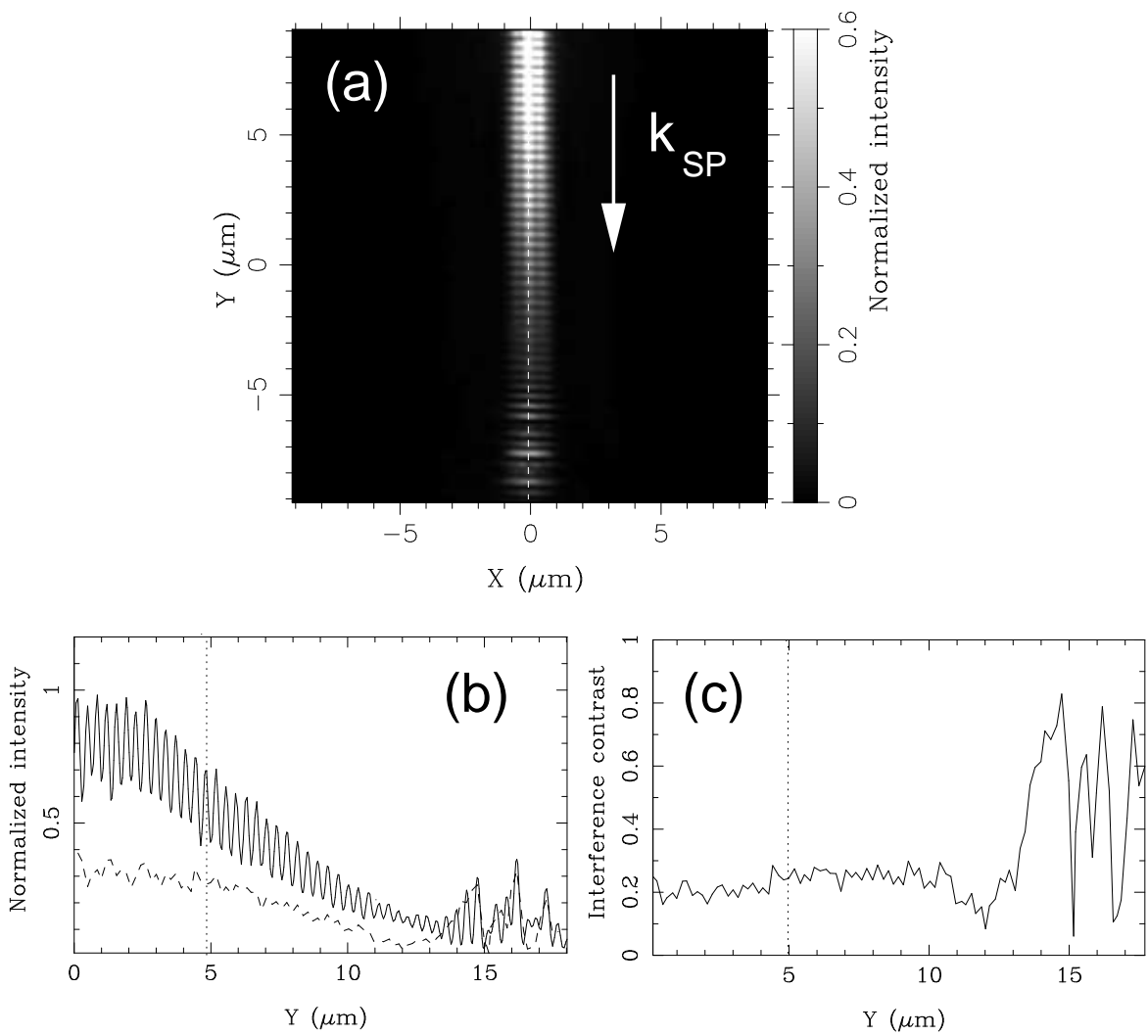

FIG. 7. (a) PSTM image of a ten slits micro-grating $(P=400 \mathrm{~nm})$. The mirror is engraved at a distance of $25 \mu \mathrm{m}$ of the SPW input. The incident wavelength is $\lambda_{0}=800 \mathrm{~nm}$. (b) (solid) Longitudinal cross-cut of the PSTM image, (dashed) Amplitude of the standing wave pattern oscillations. Note the damping of the amplitude as the observation point approaches the mirror. (c) Contrast of the oscillations in the cross-cut shown in (b). 


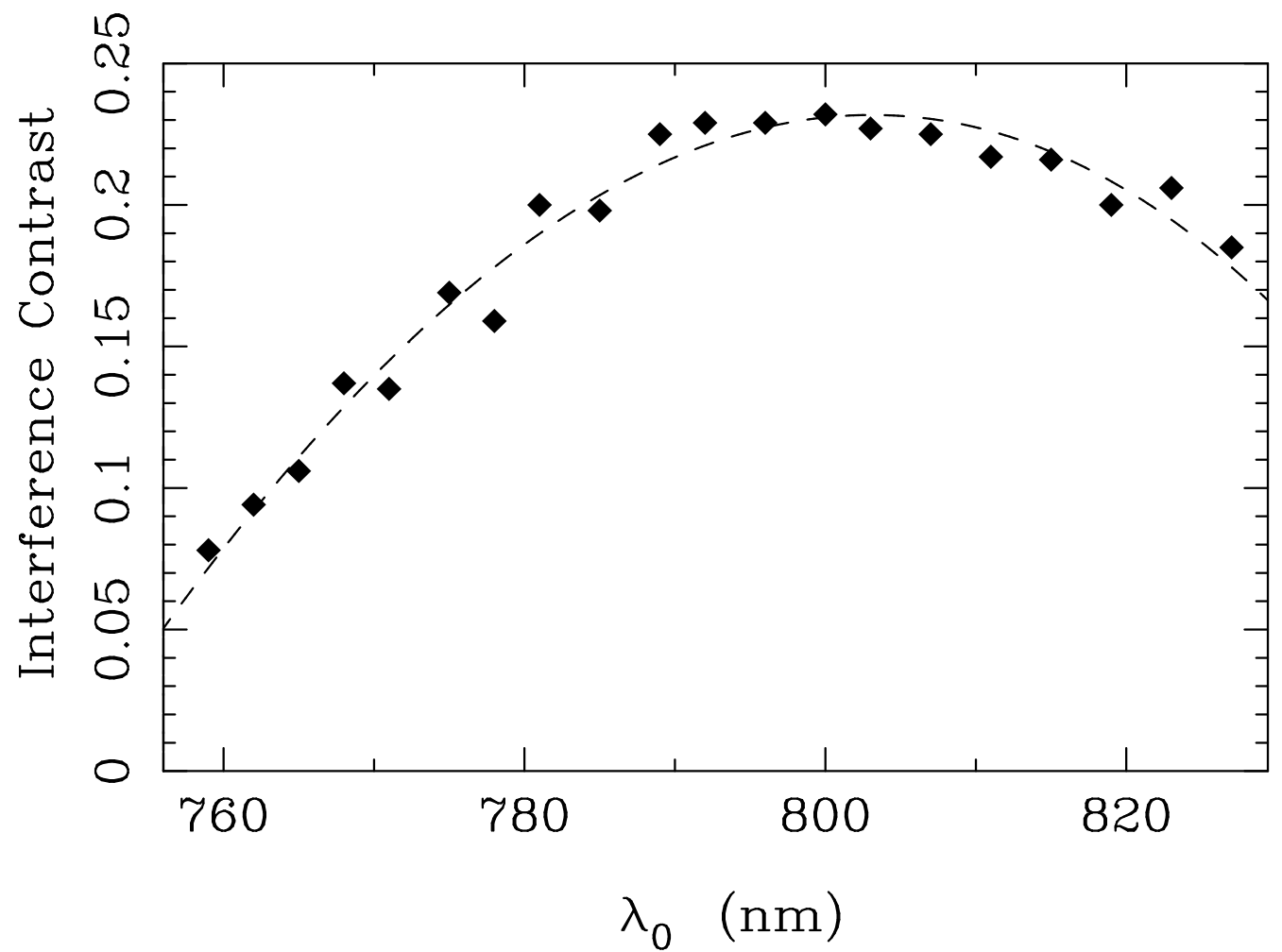

FIG. 8. Spectroscopic behavior of the standing wave pattern contrast corresponding to the PSTM images of the micro-structured SPW shown in figure 7. Dots are experimental values, the dashed line is a fit of the experimental data. 


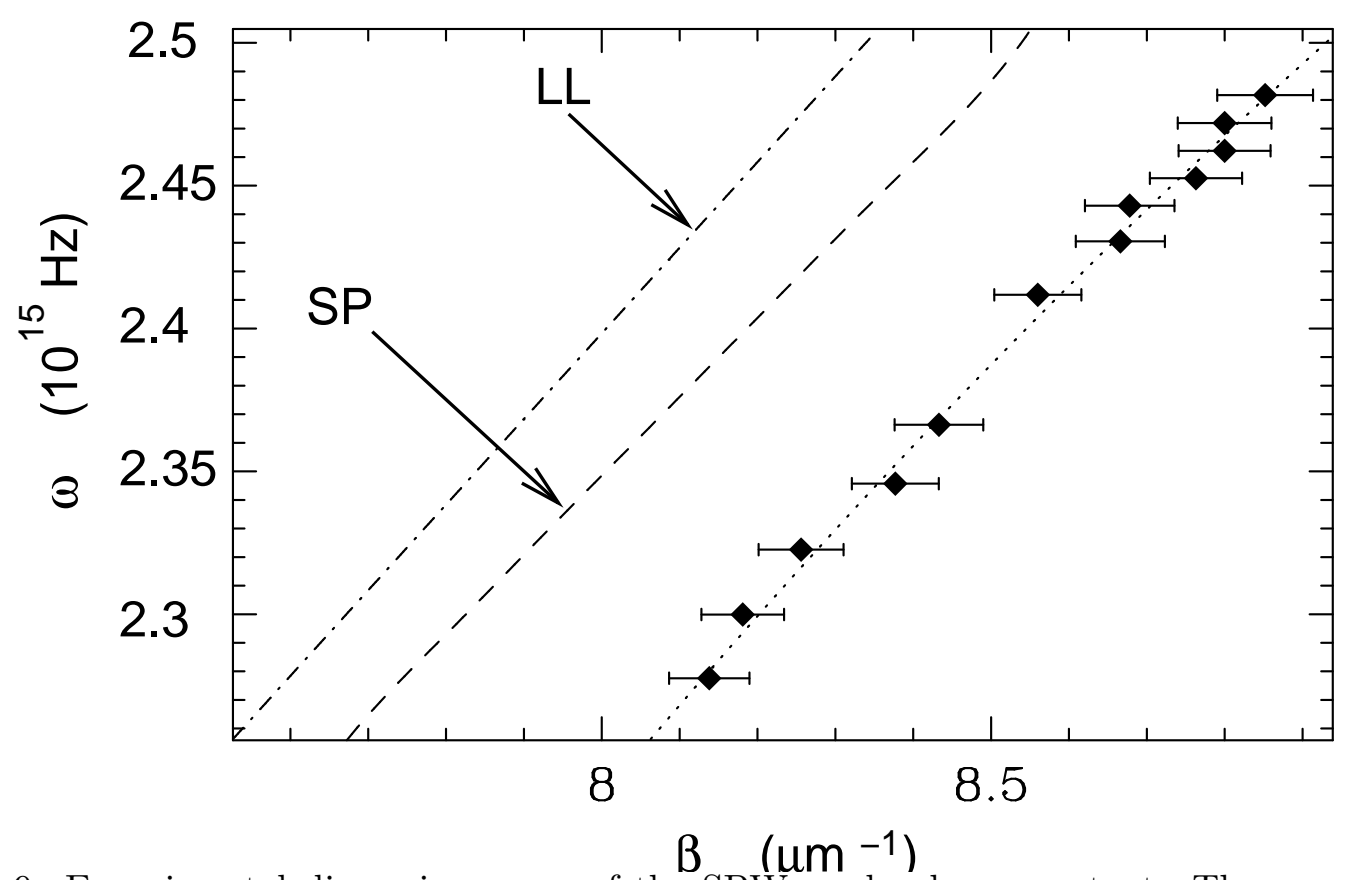

FIG. 9. Experimental dispersion curve of the SPW mode phase constant. The reported frequency range corresponds to wavelengths in vacuum varying from $760 \mathrm{~nm}$ to $830 \mathrm{~nm}$. SP (dashed): Computed dispersion curve of a surface plasmon excited at a gold/air interface. The dielectric function of gold used for the calculation of the dispersion curve has been measured by ellipsometry. LL (Dashed-dotted): Light line in air. 

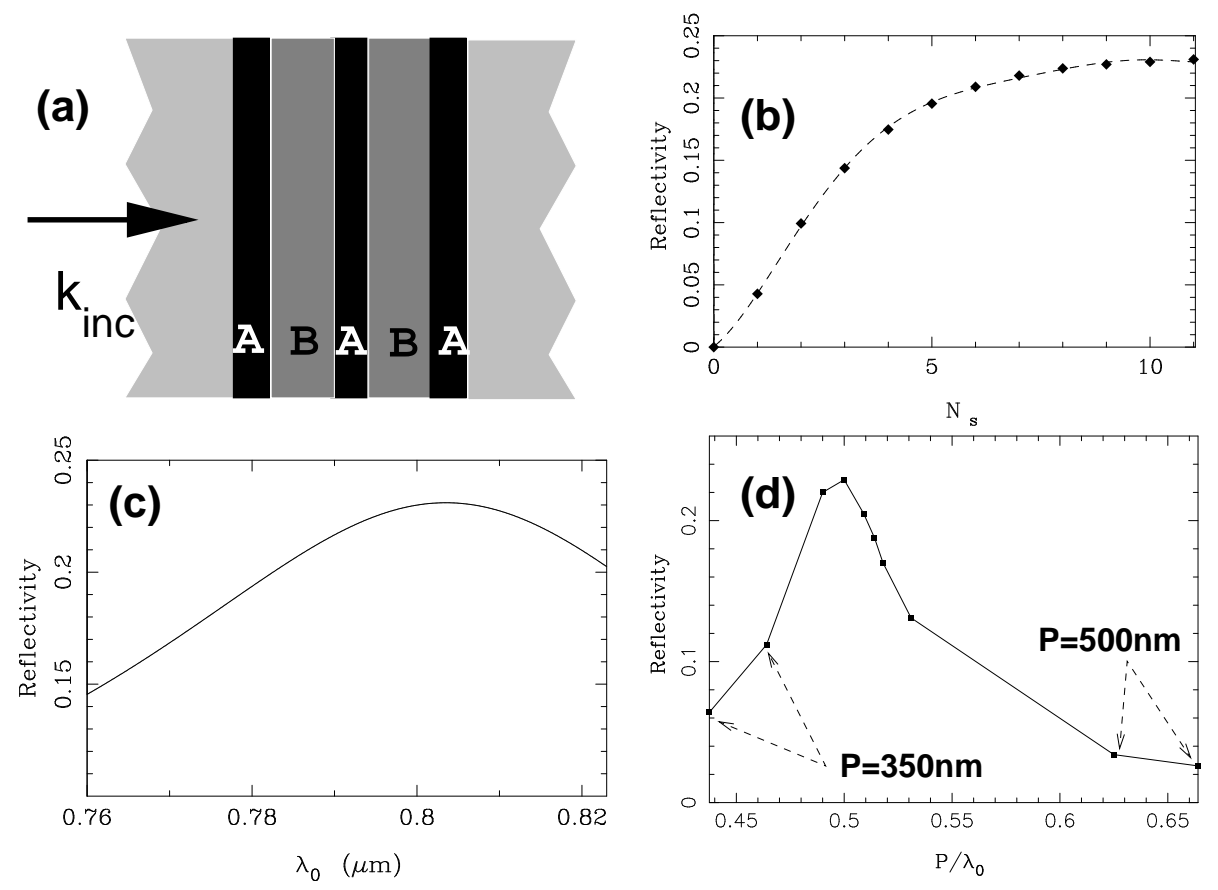
FIG. 10. (a) Scheme of equivalent Bragg Mirror (EBM) for modeling the optical properties of the SPP mirrors integrated into SPW. The EBM is made of two materials (A) and (B) modeling respectively the air gaps and the metallic parts of the micro-grating. Material (A) has been taken as an absorbing material in order to simulate the scattering losses of the incident SPW mode on the slits of the micro-grating. (b) Saturation of the EBM reflectivity for an increasing number of layers $N_{s}$. The period of the EBM is $400 \mathrm{~nm}$, the thickness of layers (A) and (B) are respectively $150 \mathrm{~nm}$ and $250 \mathrm{~nm}$ and the incident wavelength is $800 \mathrm{~nm}$ in vacuum. The indexes of the input and out media are equal to the effective index of the SPW mode (1.07). The indexes of material (A) and (B) are respectively $(1.0+\mathrm{i} 0.3)$ and 1.01. (c) Evolution of the EBM reflectivity with the incident wavelength. For this computation the EBM period is $400 \mathrm{~nm}$ and $N_{s}=10$. (d) Reflectivity of the EBM as a function of the period $P\left(N_{s}=10\right)$. 